\title{
基于全球集合预报的西北太平洋热带气旋预报的 评估和改进
}

\author{
雷荔傈 ${ }^{1}$, 葛杨金晞 ${ }^{1}$, 谈哲敏 ${ }^{*}$, 鲍旭炜 ${ }^{2}$ \\ 1. 中尺度灾害性天气教育部重点实验室, 南京大学大气科学学院, 南京 210093; \\ 2. 中国气象局上海台风研究所, 上海 200030 \\ * 通讯作者, E-mail: zmtan@nju.edu.cn
}

收稿日期：2019-10-11; 收修改稿日期：2019-10-25; 接受日期：2019-11-04; 网络版发表日期：2020-02-18

国家重点研发计划项目(编号: 2017YFC1501603)和国家自然科学基金项目(批准号: 41675052、41775057和41775064)资助

摘要 西北太平洋热带气旋在2018年7 8月间的预报, 特别是在上海登陆的温比亚(2018)、安比(2018)和云雀 (2018), 给数值模式和预报均带来巨大的挑战. 文章基于ECMWF和NCEP的集合预报对这些热带气旋的预报技巧 进行了分析. 结果表明, ECMWF的集合预报对热带气旋路径和强度的预报技巧高于NCEP的集合预报. 对于在上 海登陆的三个台风，ECMWF的集合预报对温比亚(2018)和安比(2018)的强度预报技巧优于NCEP的集合预报，但 两者对云雀(2018)在峰值附近的强度预报技巧都较低. 为了提高集合预报对热带气旋的预报技巧，文章提出了一 种集合预报成员的适应性权重估计方法. 该方法利用集合成员的预报场和分析场相对于观测的距离，适应性地估 计集合成员在集合平均中的权重。当适应性权重方法应用于ECMWF和NCEP的集合预报时，二者的集合预报对 热带气旋路径和强度的预报技巧都得到了改进, 并且ECMWF集合预报的改进要优于NCEP集合预报.

关键词热带气旋, 集合预报, 适应性权重

\section{1 引言}

在过去几十年间, 热带气旋(Tropical Cyclone, TC) 的路径预报获得了显著且连续的改进，但 $\mathrm{TC}$ 强度预报 的改进却十分有限(DeMaria等, 2014). TC的路径主要 由大尺度环境场控制, 但 TC的强度却主要由环境气流 和 TC内部的动力因素等决定. TC 的预报水平的提高主 要得益于数值天气预报模式的改进(Gopalakrishnan等, 2011)，其中包括集合预报技术(Yamaguchi等，2015)、 实时观测系统(Ruf等, 2016)、数据同化技术(Weng和
Zhang, 2016)和统计预测模式(Kaplan等，2015)等方面 的发展.

理解数值天气预报的内在可预报性和实际可预报 性有助于改进TC的路径和强度预报(Lorenz, 1963). TC 强度预报改进甚微主要是因为 TC内部动力学有限的 内在可预报性, 以及预报模式不足导致的有限的实际 可预报性. 由于 TC强度主要受其内部动力学和具有混 沌特征的湿对流控制, 因此TC强度的内在可预报性可 能低于 $\mathrm{TC}$ 路径的内在可预报性. 基于受随机动能后向 散射方案(SKEB; Berner等, 2009)和侧向边界条件扰动 
而得的集合预报，Judt等(2016)发现浴旋尺度的环流更 能抵抗向上尺度的误差增长，并且浴旋尺度环流的可 预报性主要与大尺度背景场的可预报性相连. Zhang 和Sippel(2009)发现与湿对流相关的不可观测的小初 始条件扰动可导致较大的TC生成和发展的差异. Van Sang等(2008)发现边界层很小的随机水汽扰动会带来 $\mathrm{TC}$ 强度和结构的显著变化, 这说明 $\mathrm{TC}$ 的强度预报存在 较大的内在不确定性.

在实际预报中，当水平分辨率从 $12 \mathrm{~km}$ 增加到 $1.33 \mathrm{~km}$ 时, $\mathrm{TC}$ 的强度和快速增强的预报水平得到了明 显提升(Davis等，2010). Cavallo等(2013)基于HWRF模 式的实时分析和预报发现，使用集合卡尔曼滤波数据 同化技术(EnKF; Whitaker和Hamill, 2002)可系统地减小 $\mathrm{TC}$ 的路径和强度误差，但对强 $\mathrm{TC}$ 的改进效果并不显著. 另外, 使用更精细的云解析模式、同化包括地基和机载 多普勒雷达观测等的 TC内核观测资料或应用先进的基 于集合或者混合集合——变分数据同化方法, 都可以改 善TC的强度预报(Zhang等, 2009; Aksoy等, 2013). Zhang 等(2014)利用WRF模式进行对流解析后预报, 结果表明 TC强度的实际可预报性主要受不同天气尺度的环境参 数的影响, 受热力学环境参数的影响较小.

集合预报常使用于TC可预报性的研究. Yamaguchi 等(2012，2015)利用各业务中心关于TC活动的全球中 期集合预报对多中心集合预报和单模式集合预报进行 了比较，发现多中心集合预报较最佳的单模式集合预 报具有更高的预报技巧。平均而言集合预报要优于确 定性预报，集合预报能捕捉到确定性预报漏报的 TC活 动, 并且集合预报可给出预测的概率分布. 但是, 集合 预报可被系统误差所影响，导致偏差的集合平均和不 足的集合离散度. 因此, 基于统计的集合预报后处理技 术获得了广泛的关注. 集合敷料方法(Ensemble dressing)将基于集合“最佳”成员的误差形态加入到现有的 或者调整后的集合成员之上获得新增的集合成员，从 而增加了集合数目和集合离散度(Roulston和Smith, 2003; Fortin等, 2006). 贝叶斯模型平均(Bayesian model averaging; Raftery等, 2005; Bishop和Shanley, 2008)可获 得以偏差订正后的预报为中心的、概率密度函数的加 权平均, 并且所得的贝叶斯模型平均权重系数可用于 分析集合成员的可用性. 相关的方法还包括集合密度 模式输出统计法(Ensemble kernel density model output statistics; Glahn等, 2009)和集合回归方法(Ensemble re- gression; Unger等, 2009). 值得注意的是, 集合统计后处 理方法通常需要较大的训练样本、并包含气候尺度的 信息, 因此可能不适合于TC的集合预报.

2018年7 8月西北太平洋 TC路径和强度, 特别是 其异常的路径给预报带来了具大的挑战. 一个月内三 个 TC接连登陆上海是十分罕见的, 并且强热带风暴安 比(2018)是 1949年以来第三个直接登陆上海的TC. 因 此本文的一个主要目标就是理解2018年7 8月生成和 发展于西北太平洋的 $\mathrm{TC}$ 的预报技巧. 为此, 本文对比 了ECMWF(the European Centre for Medium-Range Weather Forecasts)的集合预报系统EPS(ensemble prediction system)和NCEP(the National Centers for Environmental Prediction)的全球集合预报系统GEFS(global ensemble forecast system)对 TC 路径和强度的预报技 巧. 在此基础上，本文进一步提出了一种新的适应性 估计集合权重系数的方法，以改善 $\mathrm{TC}$ 路径和强度的集 合预报。该适应性集合权重估计方法基于集合的预报 场和分析场分别相对于观测的距离，适应性地估计集 合成员在集合平均中的权重系数; 该思想与经典粒子 滤波中的重要性采样相似(van Leeuwen, 2009). 随后 本文对此适应性集合权重系数在ECMWF和NCEP的 集合预报中的应用进行了研究.

\section{2 数据}

热带气旋要素(TCVitals)是 $\mathrm{TC}$ 参数的实时数据集, 它包含了 $\mathrm{TC}$ 的位置、最低海平面气压(SLP)、最大风 速、形状和大小等信息(Trahan和Sparling, 2012). 本文 将TCVitals所给出的TC位置和强度视为观测, 用于检 验集合预报. 本文使用ECMWF的EPS和NCEP的GEFS 的集合预报分析 TC 的预报技巧. 下面对ECMWF的 EPS和NCEP的GEFS做简要介绍.

ECMWF的EPS集合预报资料水平分辨率为 $18 \mathrm{~km}$, 垂直层数为 91 层，其每 $12 \mathrm{~h}$ 预报一次，预报时效为 $360 \mathrm{~h}$ (Magnusson等, 2019). ECMWF的EPS集合预报成员为 51 个, 其中包含一个控制成员和 50 个集合成员. 集合成 员是添加初始扰动于控制成员而得. 所添加的初始扰 动来自于集合四维变分数据同化(Ensemble of four-dimensional variational data assimilation, EDA; Bonavita 等, 2012)、奇异向量扰动(Buizza, 2008)和随机物理参 数化倾向扰动 $(\mathrm{SPPT})$ 、随机动能后向散射(SKEB)等 
随机物理方法(Leutbecher等, 2017). 为了增加 TC 路径 的集合预报离散度，奇异向量的目标区域选择在TC附 近(Barkmeijer等, 2001).

NCEP的GEFS集合预报资料水平分辨率为 $34 \mathrm{~km}$, 垂直层数为64层，其每6h预报一次，预报时效为 $192 \mathrm{~h}$ (Zhou等，2017). NCEP的GEFS集合预报成员为21个, 其中包括 1 个控制成员和 20 个集合成员; NCEP的集合 成员数目少于ECMWF. NCEP控制成员的初始条件来 自混合三维变分-集合数据同化(Hybrid three-dimensional variational-ensemble data assimilation; Kleist和 Ide，2015)，集合成员的初始条件来自于循环的集合 Kalman滤波(Ensemble Kalman Filter; Whitaker和Hamill, 2002). 另外, NCEP的集合预报使用了三种随机物 理方案, 包括SPPT(Palmer等, 2009)、SKEB(Berner等, 2009)和行星边界层水汽随机扰动方案(SHUM)等. 为 了改进TC的初始条件, NCEP的GEFS集合预报还使用 了 TC重新定位(TC relocation; Liu等, 2006), 即先将TC 从环境中分离出来并单独进行扰动, 然后将扰动后的 $\mathrm{TC}$ 加入到已经将 $\mathrm{TC}$ 移动到观测位置的初始条件中.

\section{3 方法}

基于集合的数据同化方法通常使用随机抽取的方 法获得一定数量的集合成员或者粒子，来表征模式的 概率密度函数 (probability density function; pdf). 设 $\mathbf{x}$ 为 包含所有预报变量的模式状态向量(state vector), 并设 $\mathbf{x}$ 的概率密度函数为 $p(\mathbf{x})$, 则 $\mathbf{x}$ 的平均值可通过下式 而得

$I(\mathbf{x})=\overline{\mathbf{x}}=\int p(\mathbf{x}) \mathbf{x} \mathbf{d} \mathbf{x}$.

对于基于集合的方法, $\mathbf{x}$ 的平均值可以近似为

$I(\mathbf{x}) \approx I_{N}(\mathbf{x})=\frac{1}{N} \sum_{i=1}^{N} \mathbf{x}_{i}$,

式中，下标 $i$ 代表第 $i$ 个集合成员， $N$ 代表集合成员数目. 为了得到此平均值的近似表达式， $N$ 个集合成员都是 独立、随机地从其概率密度函数 $p(\mathbf{x})$ 中抽取. 概率密 度函数 $p(\mathbf{x})$ 则可由集合成员表示为

$p(\mathbf{x})=\frac{1}{N} \sum_{i=1}^{N} \delta\left(\mathbf{x}-\mathbf{x}_{i}\right)$,

式中, $\delta$ 代表狄拉克函数. 尽管模式可为非线性的，但
EnKF通常假设预报场和分析场的概率密度函数为高 斯分布，也就是每个集合成员或粒子都有同等的概率 代表真值. 因此，每个集合成员在集合平均中的权重 都为 $1 / N$.

但是若给定一个集合预报，该集合预报中的某些 集合成员的预报结果会优于其他的集合成员(见本文 5.2 节). 如果能够给予这些拥有更好预报结果的集合 成员较大的权重，同时给予预报较差的集合成员较小 的权重，集合预报的预报技巧可能会有所改进. 因此， 本文提出了一种适应性估计集合预报中集合成员权重 系数的方法. 该适应性估计所得的权重系数可以不为 $1 / N$ ，并希望预报技巧高的集合成员获得大于 $1 / N$ 的权 重. 统计后处理方法, 如贝叶斯模型平均(Raftery等, 2005; Bishop和Shanley，2008)、集合回归方法(Unger 等，2009)和集合密度模式输出统计法(Glahn等，2009) 等, 通常依赖于大量的训练样本, 并包含气候尺度的信 息，因此这些统计后处理方法可能不适用于 TC的集合 预报. 下面将要介绍的适应性估计集合成员权重系数 的方法使用了相邻时刻的集合预报场和分析场相对于 观测的距离, 其思想相似于与经典粒子滤波中的重要 性采样(van Leeuwen, 2009).

设 $\mathbf{y}$ 为观测向量, 在给定观测 $\mathbf{y}$ 时模式状态向量 $\mathbf{x}$ 的 概率密度为

$p(\mathbf{x} \mid \mathbf{y})=\frac{1}{N} \sum_{i=1}^{N} w_{i} \delta\left(\mathbf{x}-\mathbf{x}_{i}\right)$,

其中权重系数 $w_{i}$ 为

$w_{i}=\frac{p\left(\mathbf{y} \mid \mathbf{x}_{i}\right)}{\sum_{j=1}^{N} p\left(\mathbf{y} \mid \mathbf{x}_{j}\right)}$.

式中, $p\left(\mathbf{y} \mid \mathbf{x}_{i}\right)$ 为给定模式状态向量 $\mathbf{x}_{i}$ 时观测向量 $\mathbf{y}$ 的 概率密度函数, 该函数通常认为满足高斯分布

$$
\begin{aligned}
& p\left(\mathbf{y} \mid \mathbf{x}_{i}\right)= \\
& A \exp \left\{-\frac{1}{2}\left[\mathbf{y}-H\left(\mathbf{x}_{i}\right)\right]^{\mathrm{T}} \mathbf{R}^{-1}\left[\mathbf{y}-H\left(\mathbf{x}_{i}\right)\right]\right\},
\end{aligned}
$$

式中, $H$ 是将模式状态向量 $\mathbf{x}$ 插值到观测空间的观测算 子, $\mathbf{R}$ 是观测的误差协方差矩阵, $A$ 是将 $p\left(\mathbf{y} \mid \mathbf{x}_{i}\right)$ 转化为 概率密度函数的系数.

模式状态向量 $\mathbf{x}$ 在 $t$ 时刻的预报场可由 $t-l$ 时刻的分 析场作为初始条件向前积分模式而得 
$\mathbf{x}_{i, t}^{f}=f\left(\mathbf{x}_{i, t-l}^{a}\right)$,

这里 $t$ 代表时间, $l$ 代表滞后的时间长度，上标 $f$ 和 $a$ 分别 代表预报场和分析场. 在 $t$ 时刻, 状态向量 $\mathbf{x}_{i, t}^{a}$ 为分析场, 是由基于集合的数据同化方法(如EnKF等)同化观测 $\mathbf{y}$ 后得到的. 若将分析场 $\mathbf{x}_{i, t}^{a}$ 看作是在 $t$ 时刻对系统的最 准确的估计, 然后将其作为初始条件, 将这 $N$ 个集合成 员利用模式向前积分, 则可得在未来 $t+p$ 时刻的集合预 报 $\mathbf{x}_{i, t+p}^{f}$, 这里 $p$ 表示预报时长.

此时，集合预报中各集合成员 $\mathbf{x}_{i, t+p}^{f}$ 不再具有相同 的权重，而是基于集合预报的预报场 $\mathbf{x}_{i, t}^{f}$ 和分析场 $\mathbf{x}_{i, t}^{a}$ 以及观测 $\mathbf{y}$ 获得适应性的权重系数. 该适应性集合权 重方法假设在 $t$ 时刻获得较小预报误差和分析误差的 集合成员在 $t+p$ 时刻仍能获得较好的预报. 但要注意的 是, 因为数据同化可能会含有一些随机过程, 并且预报 场 $\mathbf{x}_{i, t}^{f}$ 也不一定是由相邻的前一时刻的分析场向前积 分而得，所以具有较小预报误差的集合成员不一定为 具有较小分析误差的成员. 为了将预报误差和分析误 差都纳入考虑, 式(6)改写为

$$
\begin{aligned}
& p\left(\mathbf{y} \mid \mathbf{x}_{i, t}^{f}, \mathbf{x}_{i, t}^{a}\right)= \\
& A \exp \left\{-\frac{1}{2}\left[\mathbf{y}-H\left(\mathbf{x}_{i, t}^{f}\right)\right]^{\mathrm{T}} \mathbf{R}^{-1}\left[\mathbf{y}-H\left(\mathbf{x}_{i, t}^{f}\right)\right]\right. \\
& \left.-\frac{1}{2}\left[\mathbf{y}-H\left(\mathbf{x}_{i, t}^{a}\right)\right]^{\mathrm{T}} \mathbf{R}^{-}\left[\mathbf{y}-H\left(\mathbf{x}_{i, t}^{a}\right)\right]\right\} .
\end{aligned}
$$

基于此概率密度 $p\left(\mathbf{y} \mid \mathbf{x}_{i, t}^{f}, \mathbf{x}_{i, t}^{a}\right)$, 自适应权重 $w_{i}$ 则可 由式(5)计算而得. 使用了适应性权重系数 $w_{i}$ 的集合预 报 $\mathbf{x}_{t+p}^{f}$ 的平均值可写为

$$
I_{N}\left(\mathbf{x}_{t+p}^{f}\right)=\sum_{i=1}^{N} w_{i} \mathbf{x}_{i, t+p}^{f}
$$

适应性权重系数具有流依赖的特征，因为该系数 可随时间 $t$ 变化. 该适应性集合权重方法假设在 $t$ 时刻 拥有较小预报/分析误差的集合成员，在以 $t$ 时刻分析 场起报的集合预报中有较小的预报误差. 而 $t+p$ 时刻的 预报误差和 $t$ 时刻的预报/分析误差的时间相关会随预 报时长 $p$ 的增加而减弱, 因此适应性权重系数给集合预 报带来的影响在较短的预报时长内会更明显. 相似地, 集合预报 $\mathbf{x}_{i, t}^{f}$ 可由不同的滞后时长 $l$ 所对应的分析场向
前积分而得, 但是随着 $l$ 的增加, 集合预报 $\mathbf{x}_{i, t}^{f}$ 对适应性 权重系数的有用信息会减少. 另外, 该适应性集合权重 方法可能不适用于非线性系统，因为误差增长可能不 是线性的，并且 $t+p$ 时刻的预报误差和 $t$ 时刻的预报/分 析误差的相关性可能是非常有限的.

\section{4 案例概述}

2018年7 8月间，西北太平洋 TC的路径和强度复 杂多变, 给预报带来巨大的挑战. 本文分析了模式对 生成和发展于这个阶段的 TC的预报能力. 表1列出了 在这个阶段内的 13 个 $\mathrm{TC}$, 其中包括 3 个最大风速小于 $88 \mathrm{~km} \mathrm{~h}^{-1}$ 的热带风暴(TS)、4个最大风速小于 $117 \mathrm{~km} \mathrm{~h}^{-1}$ 的强热带风暴(STS)和 6 个最大风速大于 $118 \mathrm{~km} \mathrm{~h}^{-1}$ 的 台风(TY). $\mathrm{TC}$ 强度定级依据日本气象厅区域专业气象 中心-东京台风中心(Regional Specialized Meteorological Centre, RSMC)热带气旋强度表(Typhoon Committee, 2015)来确定. 上述 13 个 TC 的观测路径由图1所示, 其中不同颜色代表不同的强度等级. 大部分 $\mathrm{TC}$ 路径为 西北方向, 但贝碧嘉(Bebinca)和山神(Son-Tinh)向西移 动, 而悟空(Wukong)和珊珊(Shanshan)则向北移动. 如 表1和图1所示，在7 8月时间段内的 TC 具有较大变化 范围的路径和强度.

一个月内有三个风暴登陆上海是十分罕见的. 安 比(2018)于7月17日12:00UTC发展于菲律宾海, 先向北 移动并加强成为 STS，之后减弱为 TS 并于7月 22 日 04:30UTC登陆上海崇明岛. 安比(2018)是自1949年以 来第三个直接登陆上海的 TC. 相隔不到两周, 台风云 雀(2018)于 8 月 3 日登陆上海金山. 云雀(2018)的路径十 分特殊, 它先向北移动, 加强成为STS后加速向东北移 动, 然后逆时针旋转移至日本的东南方向, 并于7月 28 日16:00UTC登陆日本三重县伊势市．之后云雀(2018) 向西移动, 在日本西侧完成了两次逆时针旋转后, 最 后于8月3日02:30UTC登陆上海. 温比亚(2018)生成于 中国东海, 然后向西北移动, 于 8 月17日20:00UTC登陆 上海浦东，成为了 2018 年袭击上海的第三个TC. 三个 $\mathrm{TC}$ 在一个月的时间内连续登陆上海, 带来了大风、强 降水和洪涝等灾害, 造成了重大经济损失和社会影响. 因此本文着重分析了模式集合预报对这三个 $\mathrm{TC}$ 的预 报技巧, 以及集合预报技巧可能的提高. 
表 12018 年7 8月发展于西北太平洋的热带气旋的名称、强度等级、集合预报的起报时间和最低海平面气压 ${ }^{a}$

\begin{tabular}{|c|c|c|c|}
\hline $\mathrm{TC}$ 名称 & 等级 & 集合预报的起报时间 & 最低海平面气压(hPa) \\
\hline MARIA & TY & 7月5日00:00UTC & 915 \\
\hline SON-TINH & TS & $\begin{array}{l}\text { 7月 } 15 \text { 日 } 12: 00 \mathrm{UTC} \\
\text { 7月 } 21 \text { 日2:00UTC }\end{array}$ & 992 \\
\hline AMPIL ${ }^{*}$ & STS & 7月18日12:00UTC & 983 \\
\hline WUKONG & STS & 7月 22 日 $12: 00 \mathrm{UTC}$ & 974 \\
\hline JONGDARI $^{*}$ & TY & 7月28日 12:00UTC & 948 \\
\hline SHANSHAN & $\mathrm{TY}$ & 8月5日00:00UTC & 949 \\
\hline YAGI & TS & 8月7日12:00UTC & 983 \\
\hline LEEPI & STS & 8月 11 日 $12: 00 \mathrm{UTC}$ & 982 \\
\hline BEBINCA & STS & 8月13日00:00UTC & 982 \\
\hline RUMBIA $^{*}$ & TS & 8月 15 日00:00UTC & 978 \\
\hline SOULIK & TY & 8月20日00:00UTC & 941 \\
\hline CIMARON & TY & 8月19日00:00UTC & 940 \\
\hline JEBI & TY & 8月30日00:00UTC & 910 \\
\hline
\end{tabular}

a) 上标*表示登陆上海的热带气旋

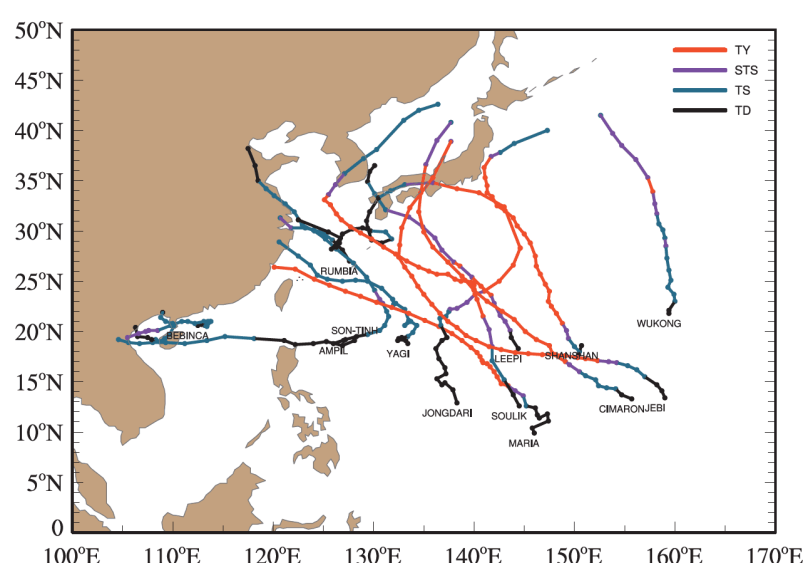

图 1 表1中 13 个热带气旋(TC)对应的观测路径 其中不同颜色代表 TC的不同强度等级

\section{5 结果分析}

\subsection{TC预报技巧分析}

为了比较ECMWF和 NCEP集合预报对 TC 路径和 强度的整体预报效果, 将表1所列的13个 $\mathrm{TC}$ 的路径和 强度集合预报按照TS、STS和TY分类并进行分别讨 论. 集合预报的起始时间如表1所示，起始时间的选择 主要基于资料的可用性，对未登陆的 $\mathrm{TC}$ 要求集合预报 包含其强度峰值, 对登陆 $\mathrm{TC}$ 要求早于其登陆五天左右. 图2为对应不同预报时长, 观测位置 $200 \mathrm{~km}$ 半径内的集 合成员 (预报误差小于 $200 \mathrm{~km}$ )占所有集合成员的平均 比值. 对每一 TC强度等级, 图 2 包含了每 $6 \mathrm{~h}$ 一次观测的
所有TC位置的观测. 图3 5的箱须图分别为 TS、STS 和TY在不同预报时长上最低海平面气压和最大风速 的预报误差的集合分布, 其中每幅图都包含了对应 TC 强度等级的TC集合预报.

分析图2中点线(TS)可得, ECMWF集合预报路径 预报误差小于 $200 \mathrm{~km}$ 的集合成员比重大于NCEP集合

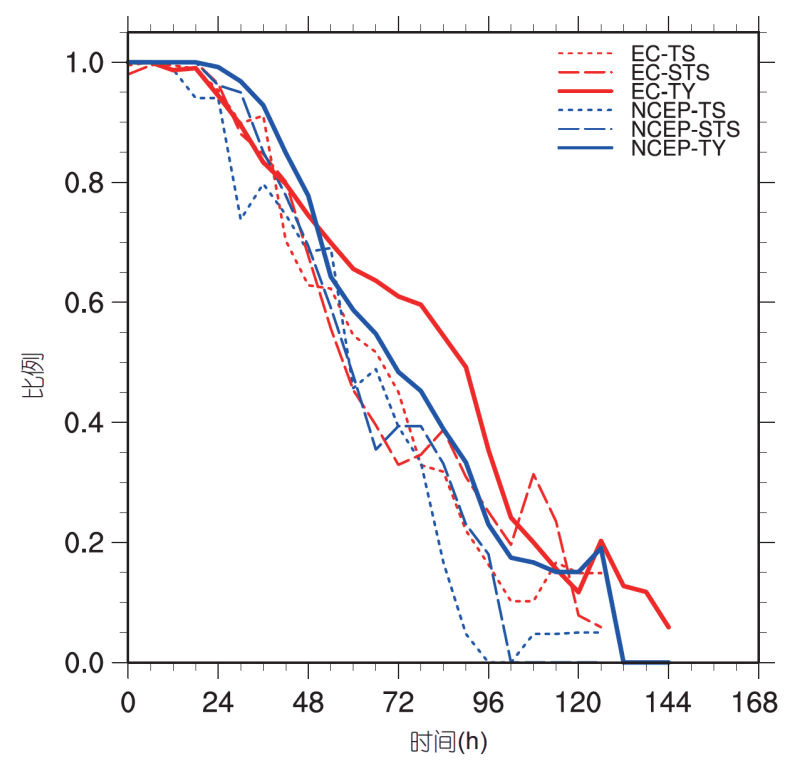

图 2 对于不同强度等级的 TC, 集合成员路径误差小于 $200 \mathrm{~km}$ 的平均占比随预报时长的变化

点线代表 TS, 虚线代表 STS, 实线代表TY, 红色为ECMWF, 蓝色为 NCEP 
预报结果，因此ECMWF集合预报对TS路径的预报技 巧略高于NCEP集合预报. 如图3所示, ECMWF集合预 报在 $30 \mathrm{~h}$ 内的最低海平面最低气压(SLP)和最大风速的 平均误差均大于NCEP，并且ECMWF集合预报所得的 强度误差分布相对于NCEP在四分位数和尾部都存在 偏差. 在 $30 \mathrm{~h}$ 之外, ECMWF集合预报的最低SLP平均误 差与NCEP相似，但ECMWF集合预报的最大风速的平 均误差小于NCEP. 当预报时效较长时, NCEP集合预报 所得的强度误差相对于ECMWF在四分位数上有更大 的偏差, 并且有更窄的尾巴, 即更小的离散度. 因此, 在 预报时长较短时，ECMWF集合预报对 TC 强度的预报 技巧低于NCEP集合预报，但在较长的预报时长时为 相反的情况.

对于STS, ECMWF和NCEP的集合预报在前 $84 \mathrm{~h}$ 预
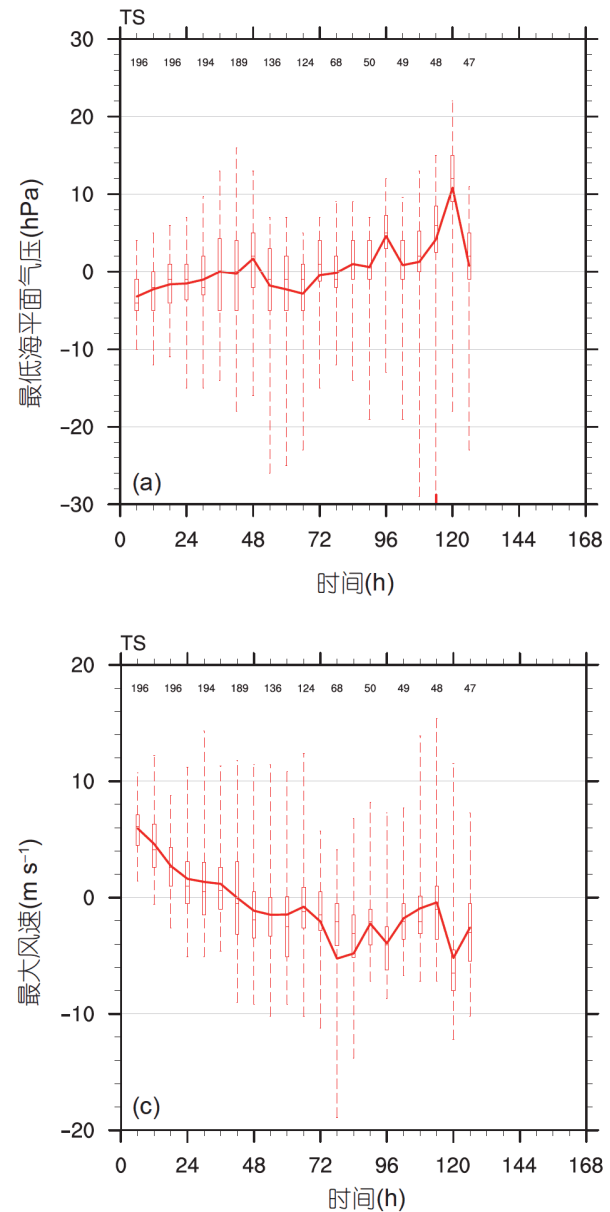

报时长内有相似的预报技巧；当预报时长超过 $84 \mathrm{~h}$ 后， ECMWF集合预报比NCEP集合预报具有更高的预报 技巧(如图2虚线所示). 如图4所示, 在24 96h, ECMWF 集合预报的最低SLP和最大风速的平均误差略小于 NCEP集合预报。平均而言，NCEP集合预报的强度误 差集合分布相对于ECMWF集合预报具有偏差更大的 四分位数和更窄的尾部(即更加不足的集合离散度). 因此, 与 TS在较长预报时效的结果相似, ECMWF集合 预报较NCEP集合预报具有更高的强度预报技巧。

对于 TY而言，当预报时长在 $24 \mathrm{~h}$ 内，ECMWF和 NCEP的集合预报对路径的预报技巧相近; 当预报时 长在24 48h时，ECMWF集合预报的路径预报技巧低 于NCEP集合预报; 而当预报时长大于 $48 \mathrm{~h}$ 后, ECMWF 集合预报的路径预报技巧高于NCEP集合预报(如图2
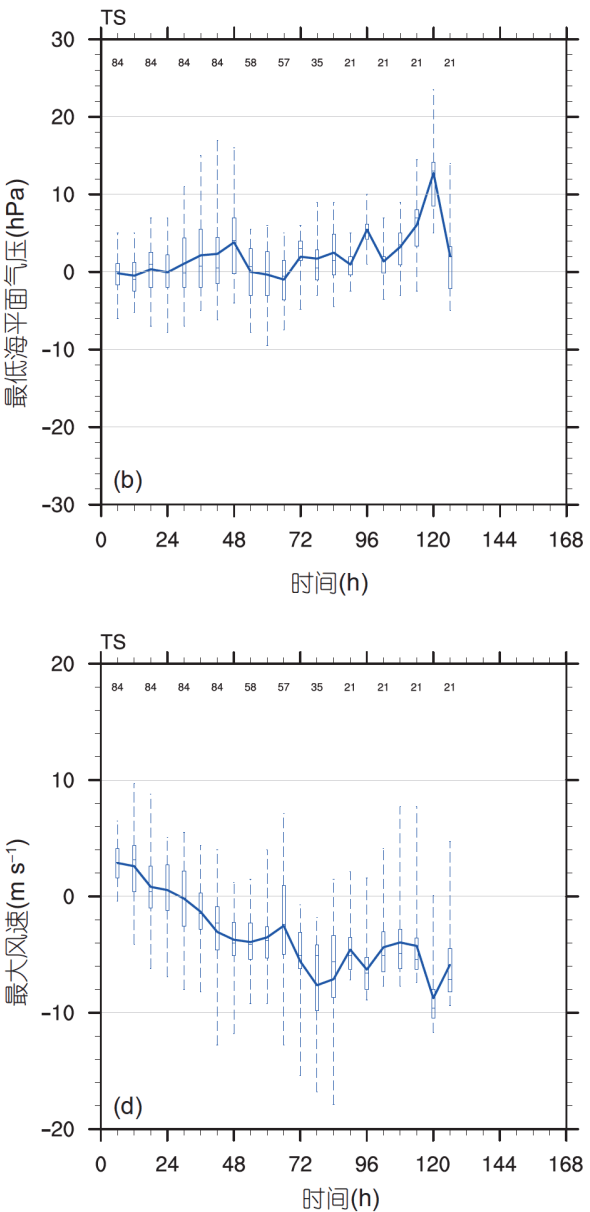

图 3 热带风暴(TS)的最低海平面气压((a), (b))和最大风速((c), (d))的预报误差的集合分布(箱须图)

左侧一列为ECMWF集合预报, 右侧一列为NCEP集合预报. 每个箱子代表集合分布的第一和第三个四分位数, 对应须部代表整个集合的范围. 实线为集合平均, 箱子内的短线为中位数 

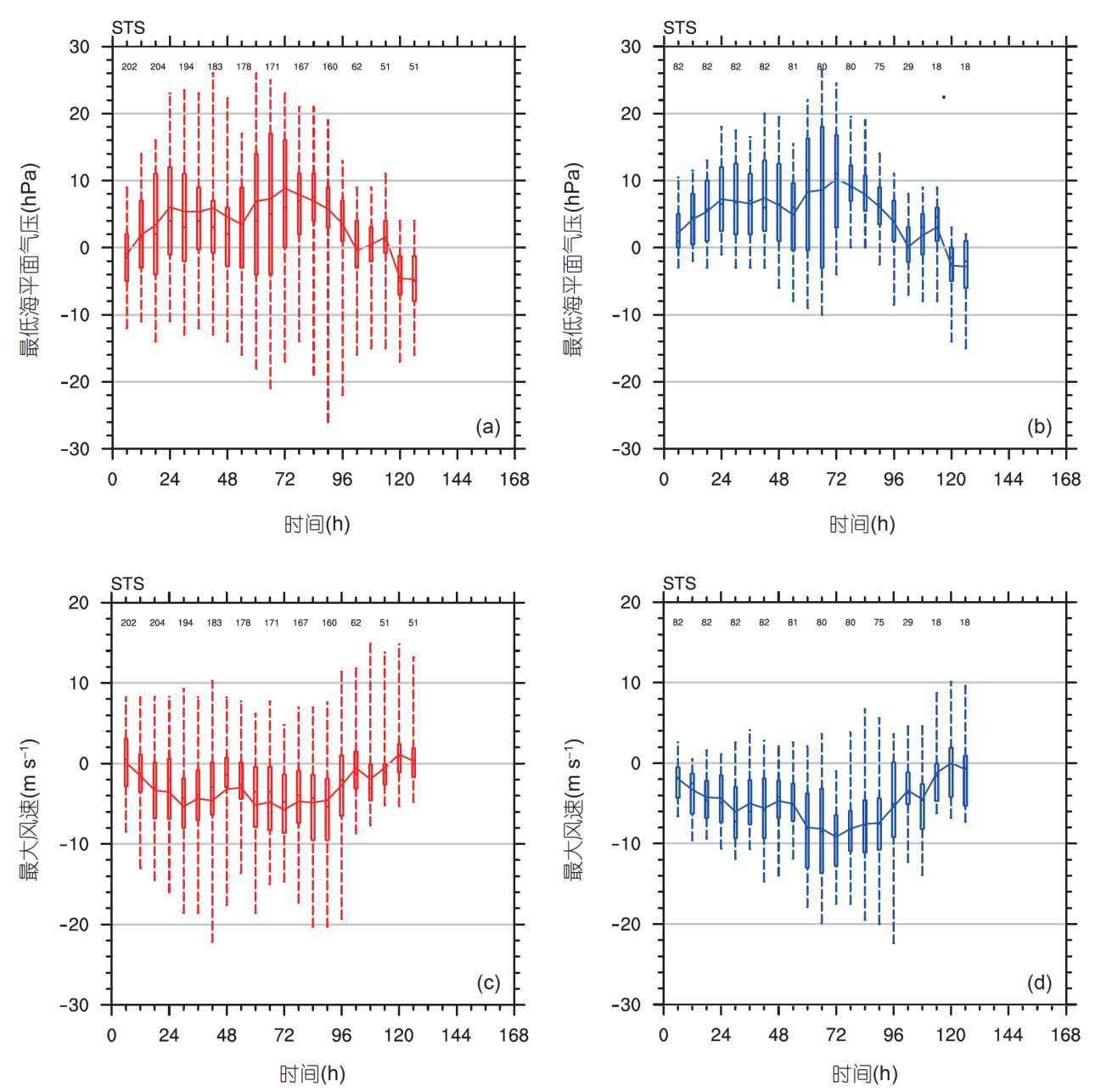

图 4 强热带风暴(STS)的最低海平面气压((a), (b))和最大风速((c), (d))的预报误差集合分布(箱须图) 说明同图3

实线所示). ECMWF集合预报所得的最低SLP和最大 风速的平均误差小于 $\mathrm{NCEP}$ (图5)。强度误差的集合成 员分布表明ECMWF集合预报相对于NCEP集合预报 有较小的四分位数和尾部的偏差. 因此, 相对于NCEP 集合预报，ECMWF集合预报具有更高的强度预报技 巧, 这与弱风暴(TS和STS)的结果是一致的.

如图2所示, ECMWF和NCEP的集合预报对强风 暴的路径预报技巧高于弱风暴，特别是在预报时效较 长时. 这可能是由于相较于弱风暴中心, 模式可更加 准确地定义强风暴中心. 图3 5中的平均误差表明, ECMWF和NCEP的集合预报通常会低估风暴强度，这 个低估随风暴增强而不断加重. 图3 5中的强度误差的 集合分布显示，随着风暴强度的增加，ECMWF和 NCEP的集合预报具有更大的四分位数间距和更长的
尾部，这表示二者对强风暴强度的预报不及弱风暴强 度的预报自信.

因此, 对于不同的风暴等级, ECMWF集合预报相 对于NCEP集合预报对 TC 路径和强度预报有更高的预 报技巧。总体而言，ECMWF和NCEP的集合预报对强 风暴的路径预报技巧高于弱风暴，但二者对强风暴的 强度预报技巧低于弱风暴.

\section{2 温比亚(2018)、安比(2018)和云雀(2018)的预 报技巧分析}

这里将对ECMWF和NCEP的集合预报对7 8月内 登陆上海的三次风暴——温比亚(2018)、安比(2018) 和云雀(2018)的预报技巧进行分析. 第3节描述的适应 性权重系数(adaptive weight, AW) 将应用于这三个风 

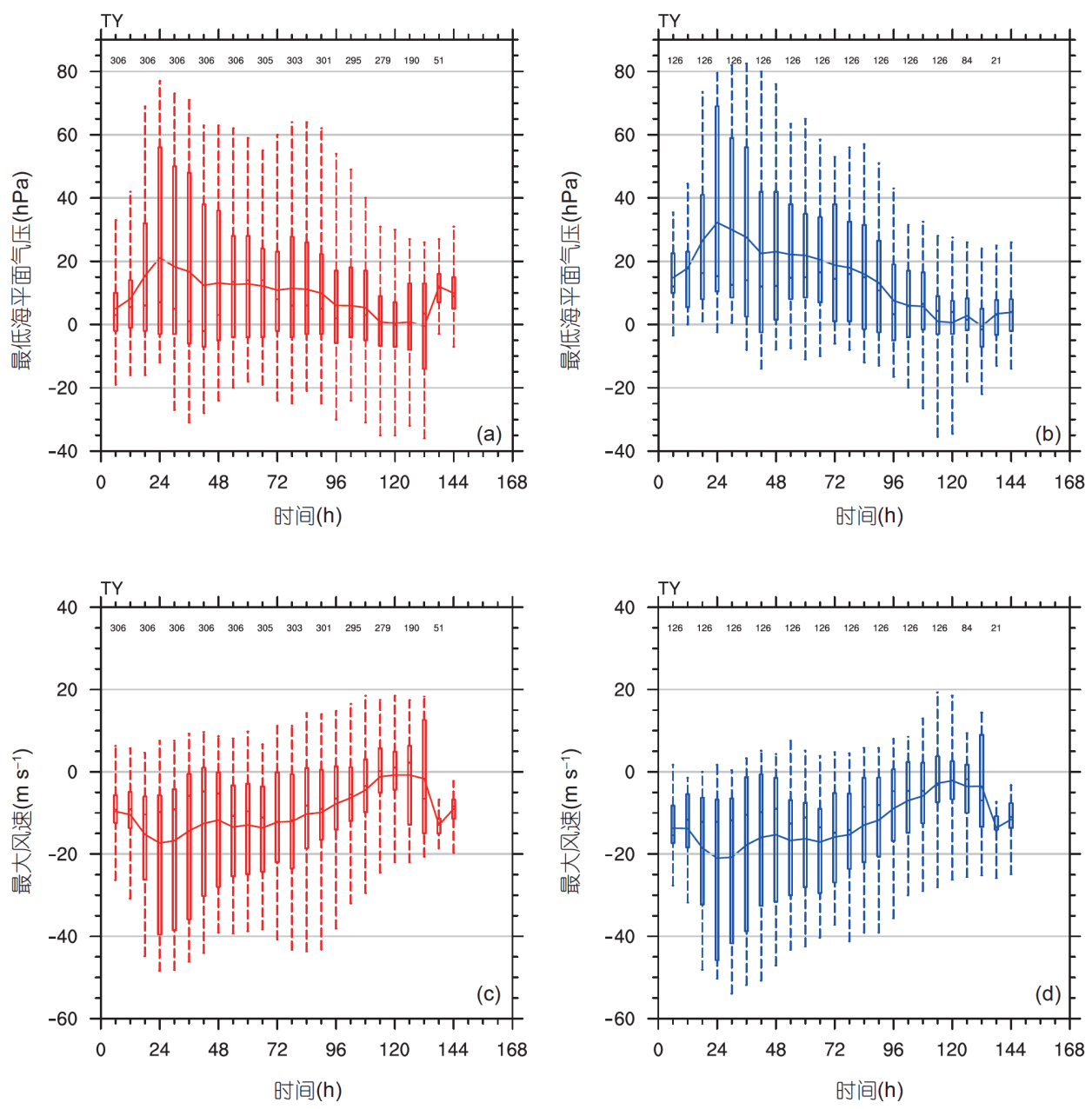

图 5 台风(TY)的最低海平面气压((a), (b))和最大风速 $((\mathrm{c}),(\mathrm{d}))$ 的预报误差集合分布(箱须图)

说明同图3

暴的ECMWF和NCEP集合预报，所得结果将与相等权 重系数(equal weight, EW)应用于ECMWF和NCEP集 合预报的结果进行对比讨论.

适应性权重系数AW可由公式(5)、(8)和(9)计算 而得. 公式(8)中的观测距离向量 $\mathbf{y}-H(\mathbf{x})$ 包含了 TC位 置、最低SLP和最大风速与观测的距离，向量维度为 $3 \times 1$. 假设观测误差之间没有相关，观测误差协方差矩 阵 $\mathbf{R}$ 为一 $3 \times 3$ 的对角矩阵. 观测误差方差, 即矩阵 $\mathbf{R}$ 对角 线上的元素，由ECMWF和NCEP对不同等级风暴的分 析误差方差分别估算而得，因此不同强度等级的风暴 所对应的观测误差方差是不同的. NCEP每天提供四 次预报，但ECMWF每天只提供两次预报，因此滞后时 间长度 $l$ 设为 $12 \mathrm{~h}$. 于是公式(8)中使用的是当前 $t$ 时刻的 分析场 $\mathbf{x}_{i, t}^{a}$ 和由 $12 \mathrm{~h}$ 前的分析场 $\mathbf{x}_{i, t-12 \mathrm{~h}}^{a}$ 起报的预
报场 $\mathbf{x}_{i, t}^{f}$

\subsection{1 温比亚(2018)}

图6给出了 TS温比亚(2018)的路径、最低SLP和 最大风速的集合预报. 在每张图中, 细粉线和粗红线分 别表示ECMWF集合预报的集合成员和集合平均值， 细浅蓝线和粗蓝线分别表示NCEP集合预报的集合成 员和集合平均值, 黑线则代表观测.

ECMWF和NCEP的集合预报都能大致捕捉温比 亚(2018)的路径. ECMWF和NCEP集合预报的路径预 报平均值相似, 且两者都与观测接近. 因此, ECMWF 和NCEP集合预报对温比亚(2018)的路径预报有较好 的预报技巧。但是，ECMWF和NCEP的集合预报未能 达到温比亚(2018)的最低SLP和最大风速的实际观测 


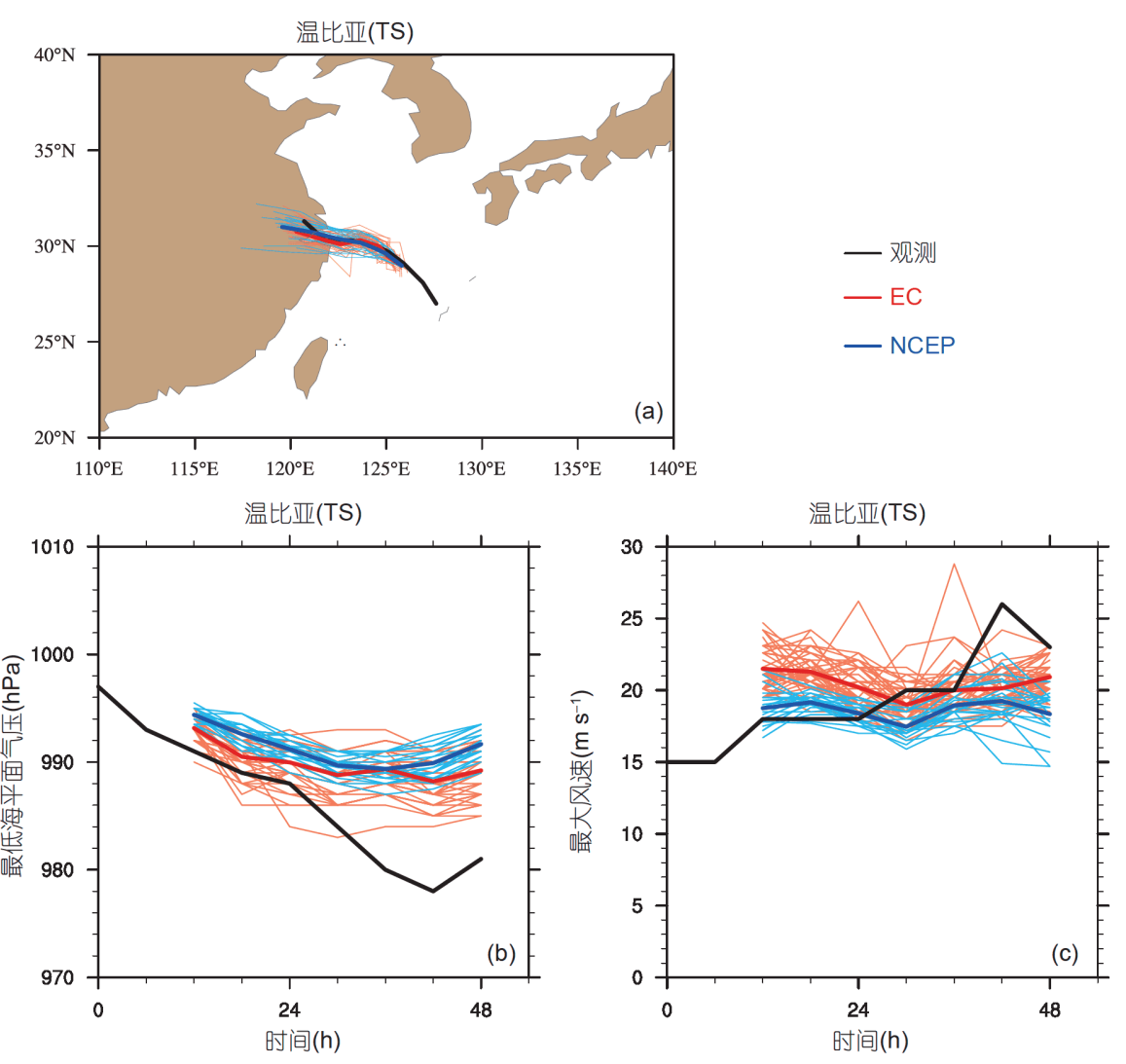

图 $6 \mathrm{ECMWF}$ (红色)和 NCEP(蓝色)于8月15日00:00UTC起报的温比亚(2018)集合预报结果

黑色为观测, (a)为路径, (b)为最低SLP, (c)为最大风速. 加粗的红线和蓝线代表集合平均

值，只有少数ECMWF集合预报的成员靠近观测的最 大风速(图6b和6c). ECMWF的集合平均相对于NCEP 的集合平均具有更小的最低SLP值和更大的最大风速 值，并且ECMWF的强度预报误差的集合分布与NCEP 相似. 因此，ECMWF集合预报对温比亚(2018)的强度 预报技巧要略高于NCEP集合预报.

图7展示了分别使用EW和AW的ECMWF和NCEP 集合预报的平均值、第一和第三个四分位数. 对于分 析场和6h预报，ECMWF集合预报使用AW比使用 EW 整体而言获得了对路径、最低SLP和最大风速的更小 的平均误差、偏差和四分位数间距; 但使用 $\mathrm{AW}$ 和 $\mathrm{EW}$ 所得结果的区别随预报时效的增长而减小. NCEP集合 预报使用 $\mathrm{AW}$ 相比于使用 $\mathrm{EW}$ 的差别很小. NCEP集合 预报使用AW和EW所得的强度集合预报相似，除了在 几个预报时长上使用 $\mathrm{AW}$ 所得的四分位数间隔减小和 偏差略微减小. 因此，AW的应用提高了ECMWF集合 预报在短预报时效上的预报技巧.

\subsection{2 安比(2018)}

STS安比(2018)集合预报的细面图如图8所示. ECMWF集合预报的集合成员在路径上向南偏移，因 此其集合平均的路径也偏南. 与ECMWF集合预报相 比，NCEP集合预报能更好地捕捉观测路径，尽管其大 多数集合成员在较长预报时效也有路径的南偏. ECMWF和NCEP集合预报的平均路径预报的登陆点 相对于实际观测都偏南. 如图 $8 \mathrm{~b}$ 和 $8 \mathrm{c}$ 所示, ECMWF集 合预报对最低SLP和最大风速做出了较好的预测, 其 集合平均值与观测也基本一致. 然而NCEP集合预报 的集合成员和集合平均都低估了最低SLP和最大风速. 虽然NCEP集合预报对安比(2018)的路径预报技巧高 于ECMWF，但ECMWF的集合预报对强度的预报技巧 高于NCEP. 因此对于安比(2018), 不同中心的集合预 报对其路径和强度的预报技巧并不一致.

对于安比(2018), ECMWF和NCEP的集合预报分 别使用 $\mathrm{EW}$ 和 AW所得的平均值、第一和第三个四分 

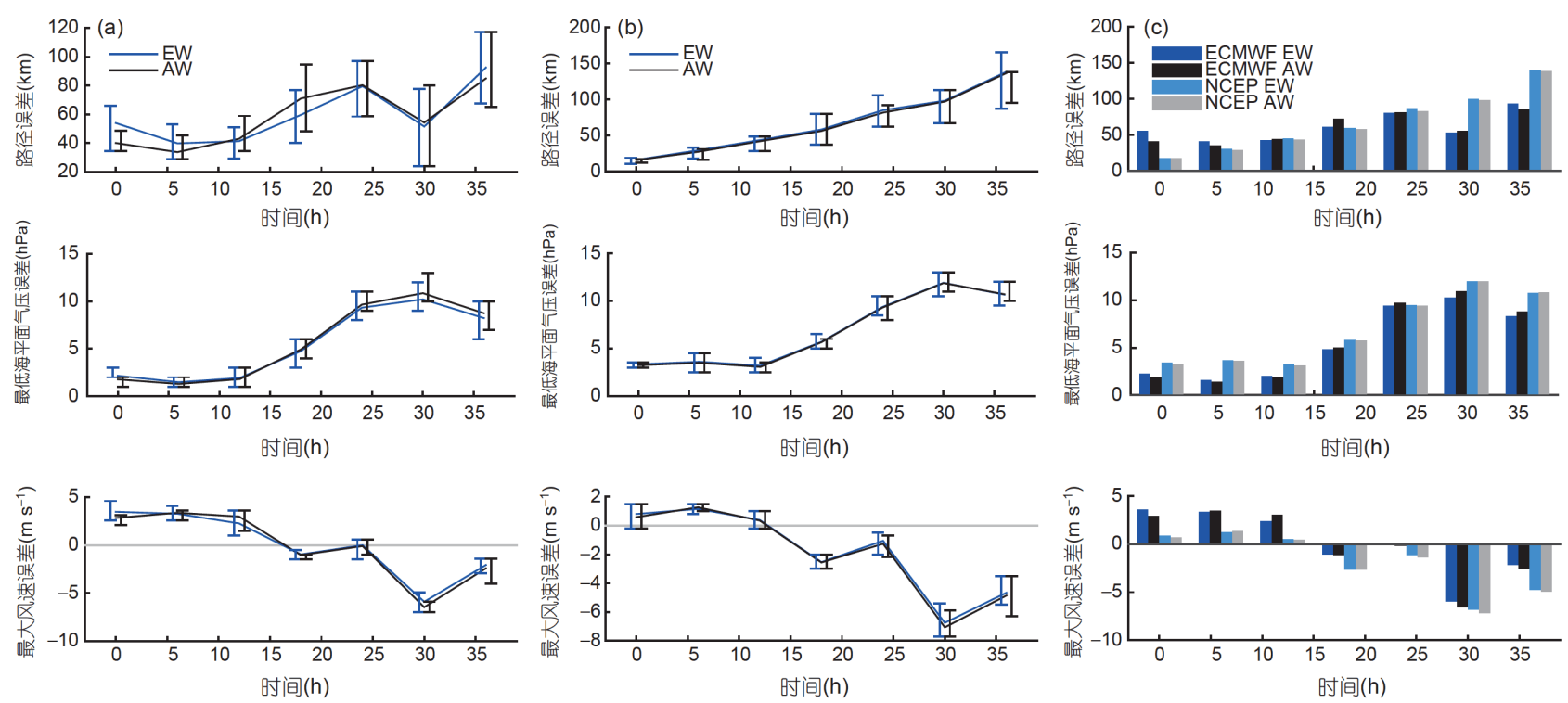

图 7 对于温比亚(2018), ECMWF(a)和NCEP(b)集合预报的集合误差分布, 以及ECMWF和NCEP的集合平均误差(c) 其中第一行为路径误差, 第二行为最低海平面气压误差, 第三行为最大风速误差. (a)和(b)中的短线代表第一和第三个四分位数. 蓝色代表相等 权重系数(EW)所得的误差, 黑色代表适应性权重系数(AW)所得的误差
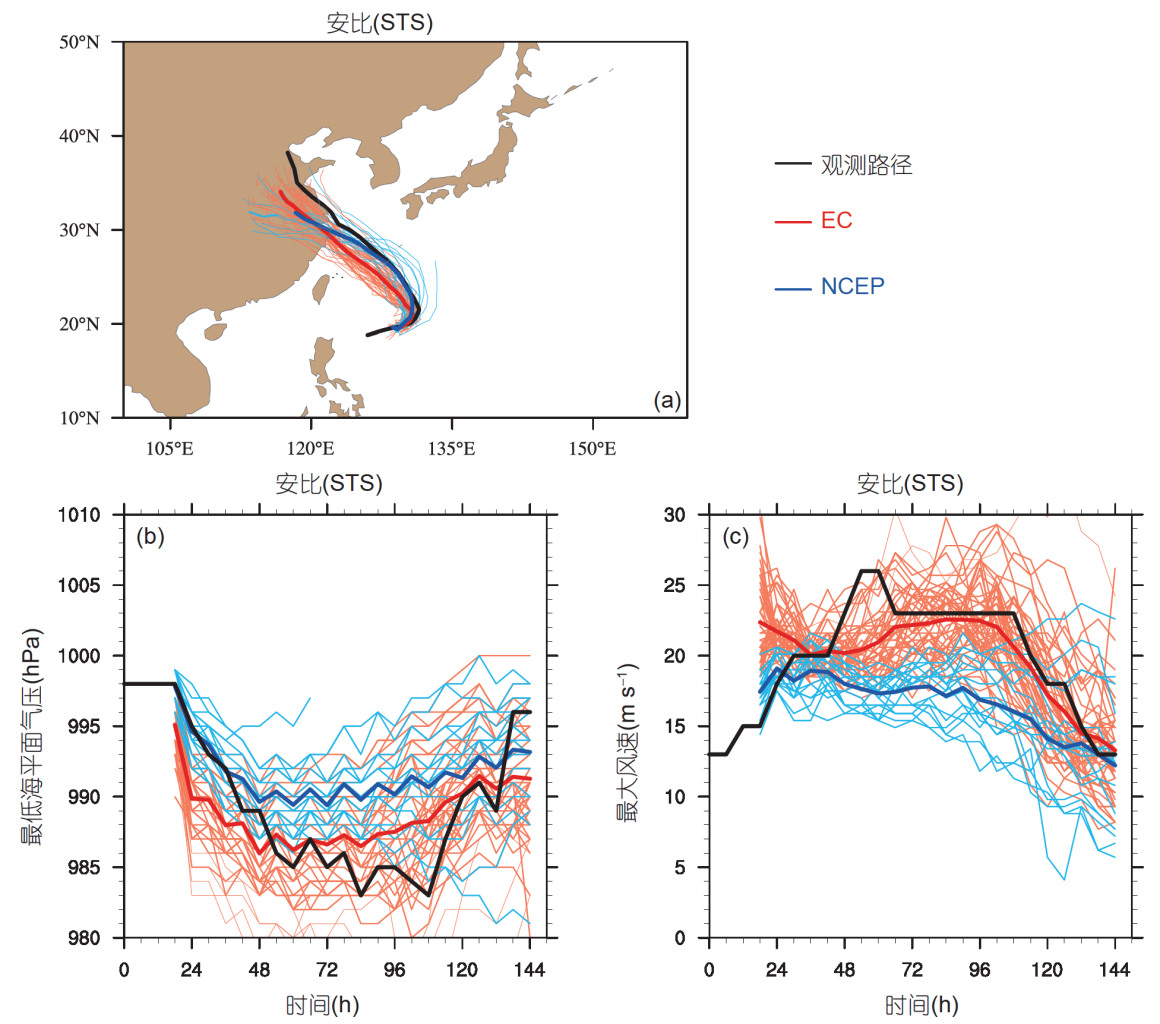

图 8 ECMWF(红色)和NCEP(蓝色)于7月18日12:00UTC起报的STS安比(2018)集合预报结果

说明同图6

位数如图9所示. 总体而言, ECMWF集合预报使用AW 相对于使用EW所得的路径平均误差更小, 并且所得的
四分位数的偏差也更小. 使用AW相对于EW在路径预 报的优势一直持续到 $144 \mathrm{~h}$ 的预报时长. ECMWF集合 
预报使用 $\mathrm{AW}$ 和使用EW有相似的最低SLP误差，但使 用AW比使用EW所得的四分位数间隔更窄. 对于最大 风速，ECMWF集合预报使用AW相对于使用EW所得 的的平均误差更小、四分位数宽度更窄且偏差更小. 因此对于安比(2018), 整体而言使用 AW 可提高 ECMWF集合预报对路径和强度的预报技巧。

总的来说，NCEP集合预报使用AW比使用EW所 得的路径的平均误差更小、四分位数宽度更窄. 应用 AW 对NCEP集合预报的最低SLP和最大风速的平均误 差影响很小, 除了会造成强度平均误差的略微增加. 但 NCEP集合预报使用AW所得的强度的四分位数宽度 要远小于EW，这意味着应用 $A W$ 使得NCEP集合预报 更加自信. 这一方面是因为适应性权重系数主要集中 于少数几个集合成员; 另一方面, NCEP集合预报也仅 包含了 20 个集合成员. 综上, AW的应用提高了 NCEP 集合预报对安比(2018)的路径预报技巧，但并未明显 提高强度的预报技巧.

\subsection{3 云雀(2018)}

台风云雀(2018)先登陆了日本三重县伊势市，然 后又登陆上海金山。本文首先分析了在云雀(2018)登 陆伊势市之前，于7月24日12:00UTC起报的集合预报. 图10为台风云雀(2018)的路径和强度的集合预报。对 于路径的预报，ECMWF集合预报有向西的偏移，而
NCEP集合预报却有向东的偏移. ECMWF和NCEP的 集合预报都预测到了路径向西的转向，但NCEP集合 预报在末期给出了再向东转向的预测. ECMWF集合 预报低估了云雀(2018)的最低SLP和最大风速, 但 NCEP集合预报相对于ECMWF集合预报更加低估了 云雀(2018)的强度，尽管NCEP集合预报的一个成员比 其他成员更加接近观测. 因此, 于7月24日12:00UTC时 起报的ECMWF和NCEP集合预报对云雀(2018)的路径 和强度的预报技巧都较低.

图11给出了云雀(2018)于7月24日12:00UTC时起 报的集合预报分别使用 $\mathrm{AW}$ 和 $\mathrm{EW}$ 时所得的平均值、 第一和第三个四分位数. 对于云雀(2018)的路径、最 低SLP和最大风速，ECMWF集合预报使用AW比使用 $\mathrm{EW}$ 获得了更小的平均误差、更窄的四分位数宽度和 更小的偏差. 因此, AW 的应用相对于 EW 改进了 ECMWF集合预报对路径和强度的预报。相似地, $\mathrm{NCEP}$ 集合预报使用 AW比使用EW也获得了对云雀 (2018)路径、最低SLP和最大风速更小的平均误差、

更小偏差的四分位数宽度. 因此应用 $\mathrm{AW}$ 于7月24日 12:00UTC起报的ECMWF和NCEP集合预报比使用 $\mathrm{EW}$ 可提高云雀(2018)路径和强度的预报技巧.

现对台风云雀(2018)在登陆上海金山之前，于7月 28日12:00UTC起报的集合预报进行分析. 如图12所示， $\mathrm{NCEP}$ 集合预报的大多数集合成员都预测出了云雀
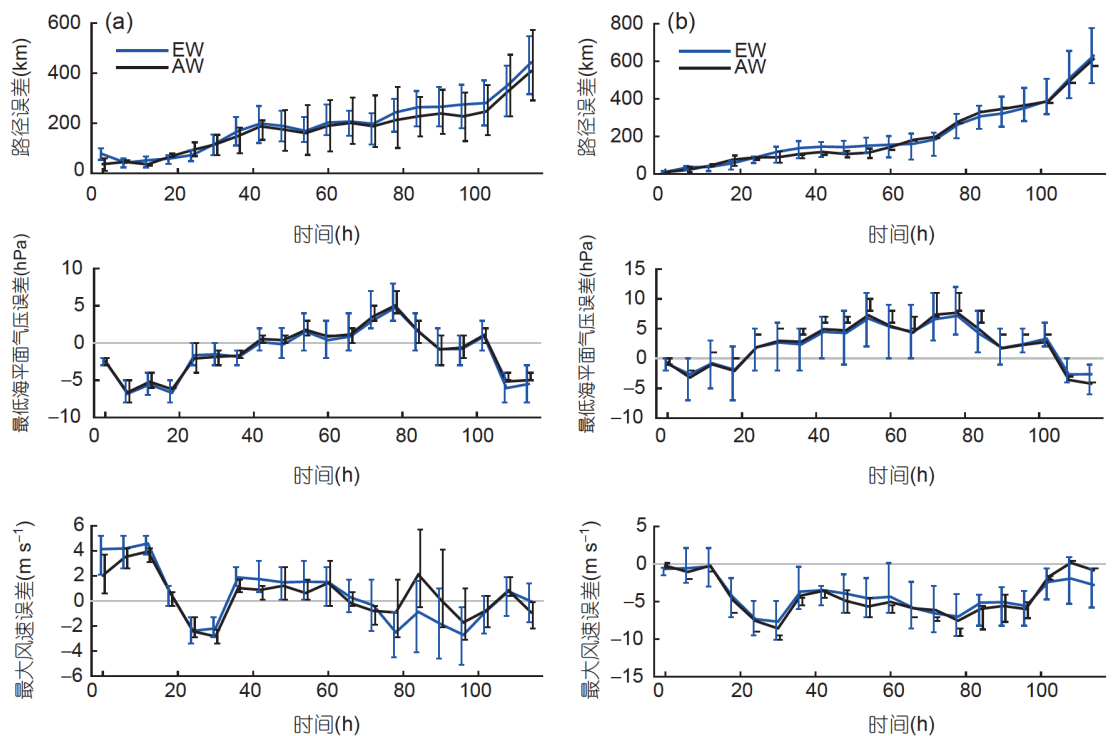
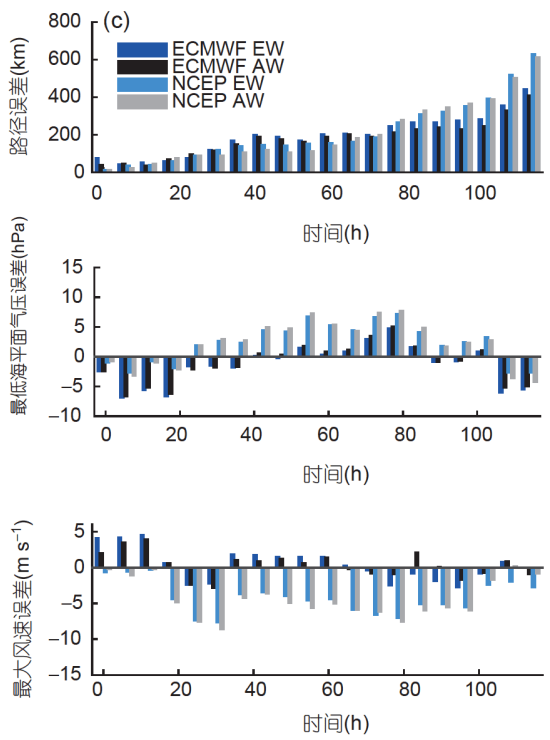

图 9 对于安比(2018), ECMWF(a)和NCEP(b)集合预报的集合误差分布, 以及ECMWF和NCEP的集合平均误差(c) 起报时间为7月18日12:00UTC 

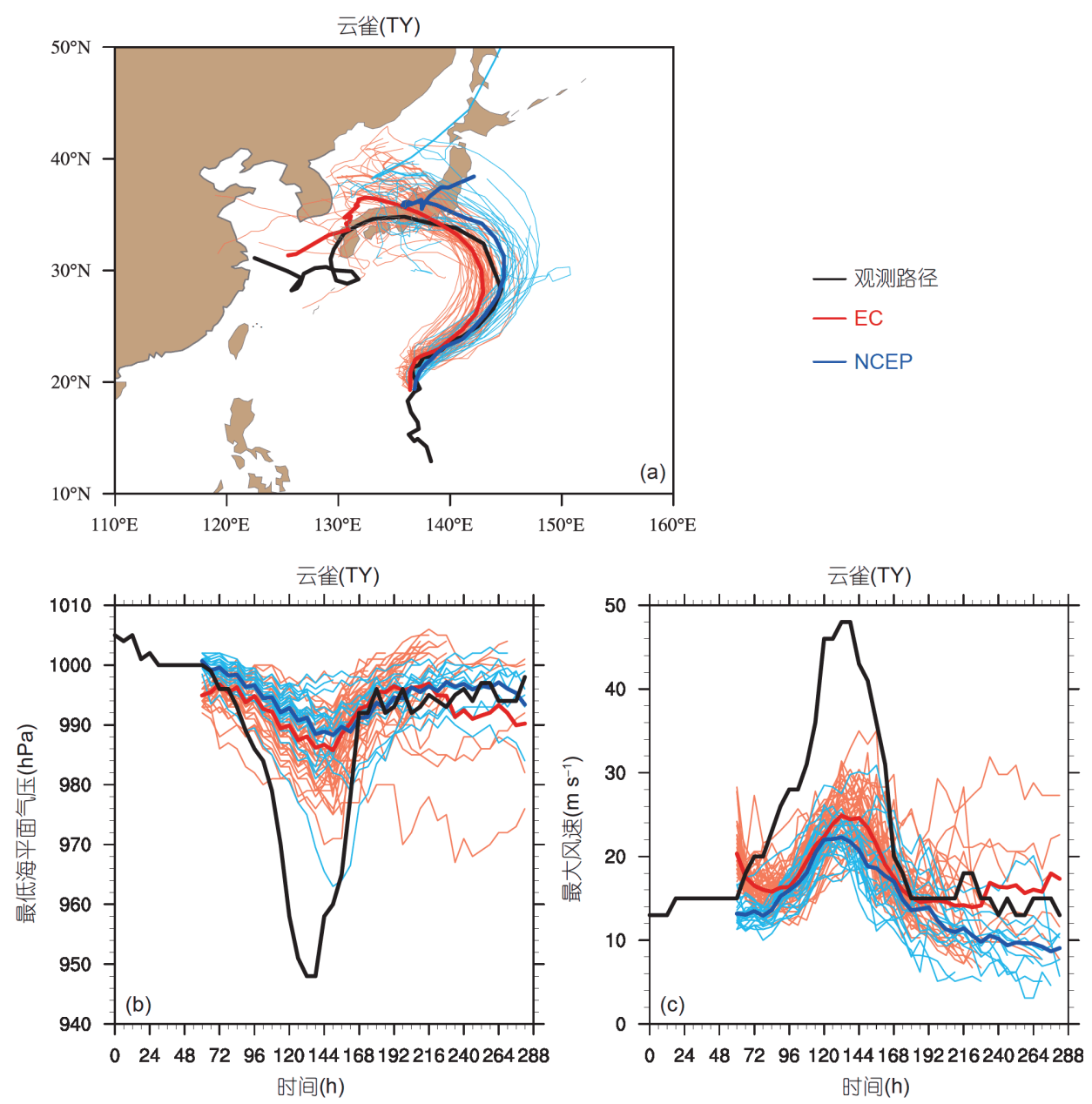

图 $10 \mathrm{ECMWF}$ (红色)和NCEP(蓝色)于7月 24日12:00UTC起报的云雀(2018)集合预报结果 说明同图6

(2018)在日本西南部第一次异常的路径转向，但没有 成员预测到其第二次转向. 相比之下, ECMWF集合预 报却没有一个成员捕捉到云雀(2018)的这两次异常路 径转弯. 与观测相比, ECMWF和NCEP的集合预报都 有向北偏移的路径，但它们正确预报了其登陆位置. ECMWF和NCEP集合预报对台风强度减弱的过程给 出了较好的预报, 但ECMWF集合预报对云雀(2018)减 弱后的强度估计大于观测和NCEP集合预报(图12b和 12c). 因此，对于台风云雀(2018)的在减弱阶段的路径 和强度预报，于7月28日12:00UTC起报的NCEP集合预 报的预报技巧高于ECMWF集合预报.

图13给出了于7月28日12:00UTC起报的集合预报 分别使用AW和EW所得的云雀(2018)的平均值、第一 和第三个四分位数. ECMWF集合预报使用 $\mathrm{AW}$ 和 $\mathrm{EW}$
所得的路径预报误差相似，但当预报时长大于 $84 \mathrm{~h}$ 时， 使用 $\mathrm{AW}$ 得到的平均路径误差稍大于使用 $\mathrm{EW}$ 所得的 平均路径误差. 对于最低SLP，ECMWF集合预报使用 $\mathrm{AW}$ 所得的误差和四分位数偏差均小于使用 $\mathrm{EW}$ 所得 的误差和四分位数偏差. 对于最大风速, ECMWF集合 预报使用 AW 和EW所得的误差相似. NCEP集合预报 使用 $\mathrm{AW}$ 比使用 $\mathrm{EW}$ 所得的路径预报的平均误差更 小、四分位数宽度也更窄，但对于强度AW和EW两种 方法所得的误差相似。因此, AW 的应用提高了 ECMWF和NCEP于7月28日 12:00UTC起报的集合预报 对云雀(2018)路径的预报技巧.

由以上分析可见, 对于登陆上海的三次风暴, 整体 而言，NCEP集合预报对路径的预报技巧高于ECMWF 集合预报，但ECMWF集合预报对强度的预报技巧高 

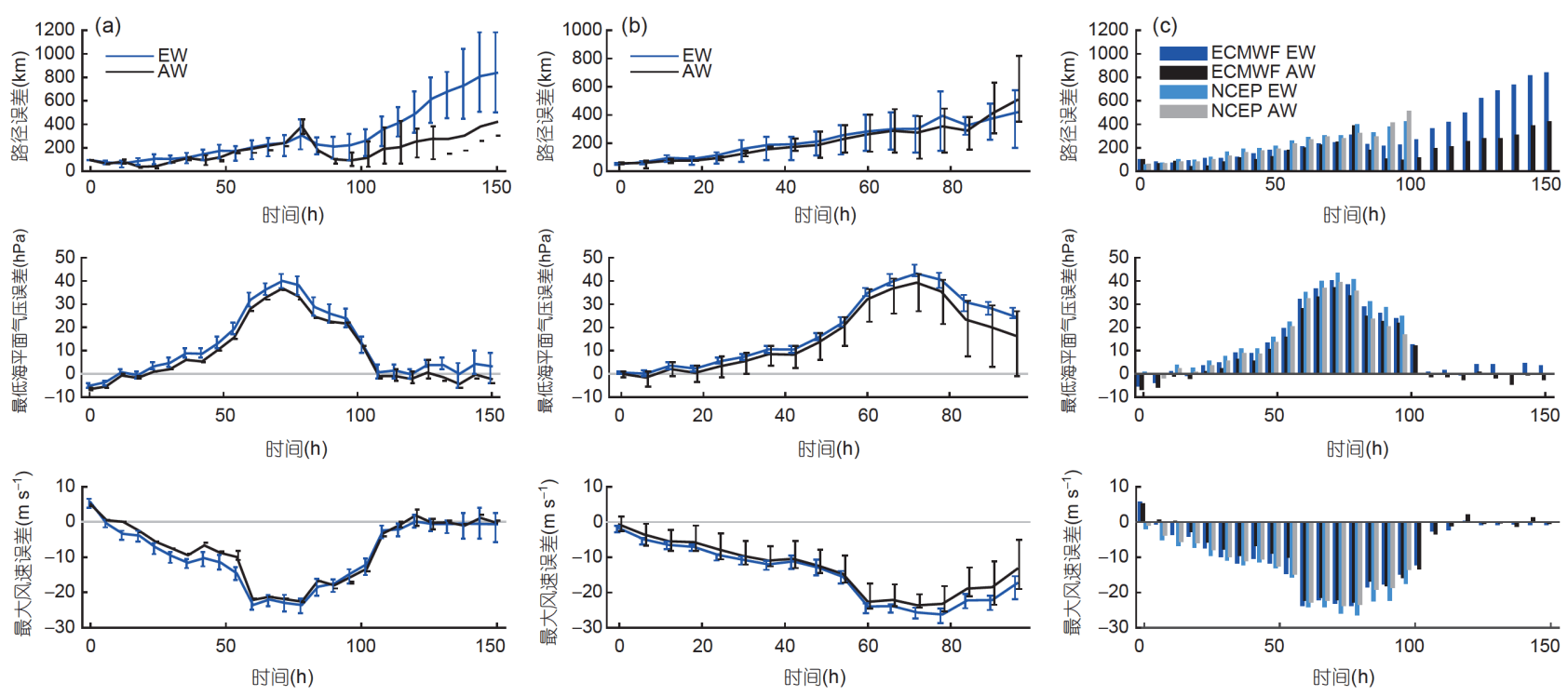

图 11 对于云雀(2018), ECMWF(a)和NCEP(b)集合预报的集合误差分布, 以及ECMWF和NCEP的集合平均误差(c) 起报时间为7月24日12:00UTC
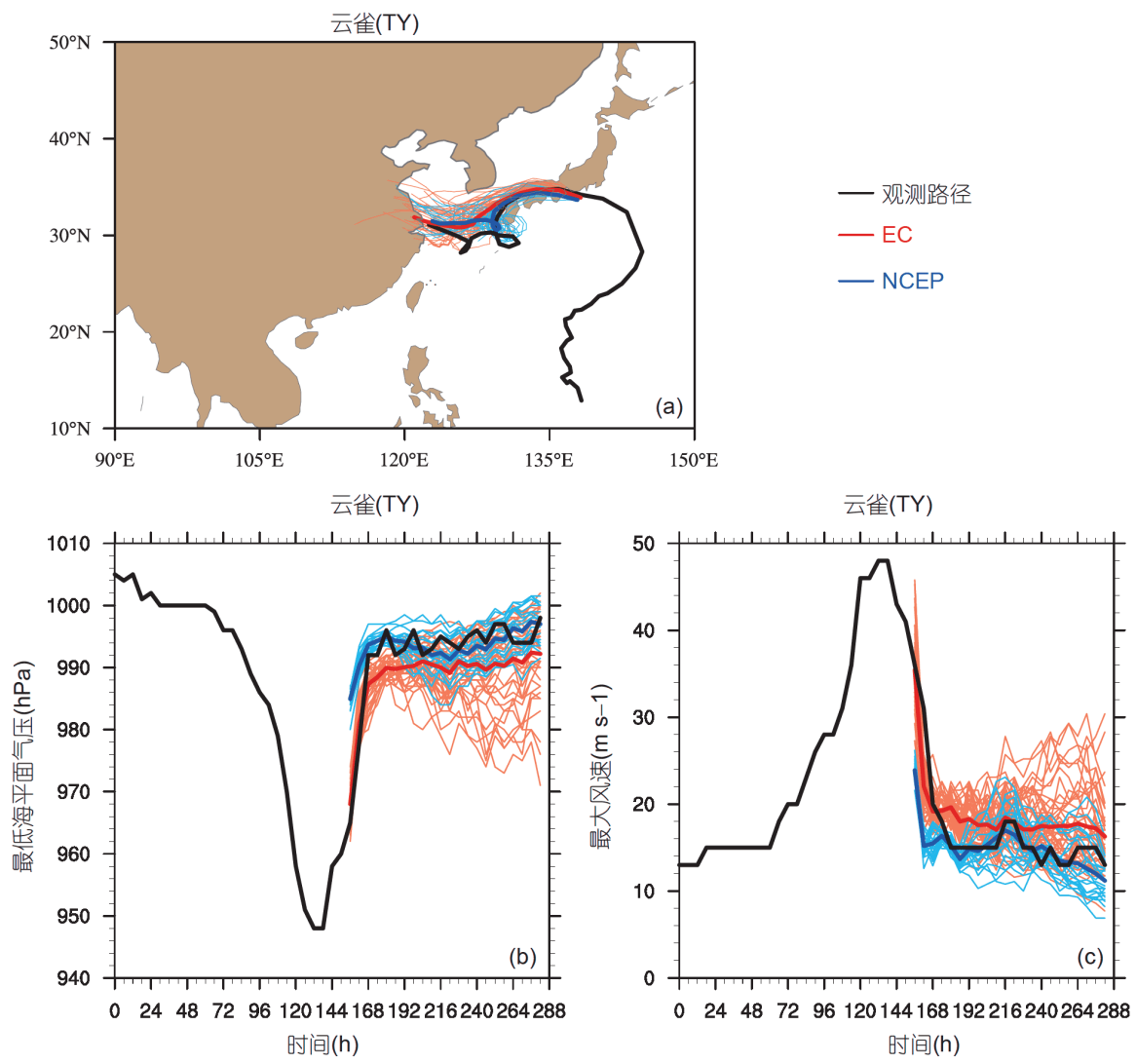

图 $12 \mathrm{ECMWF}$ (红色)和NCEP(蓝色)于7月 28日12:00UTC起报的云雀(2018)集合预报结果

说明同图6 

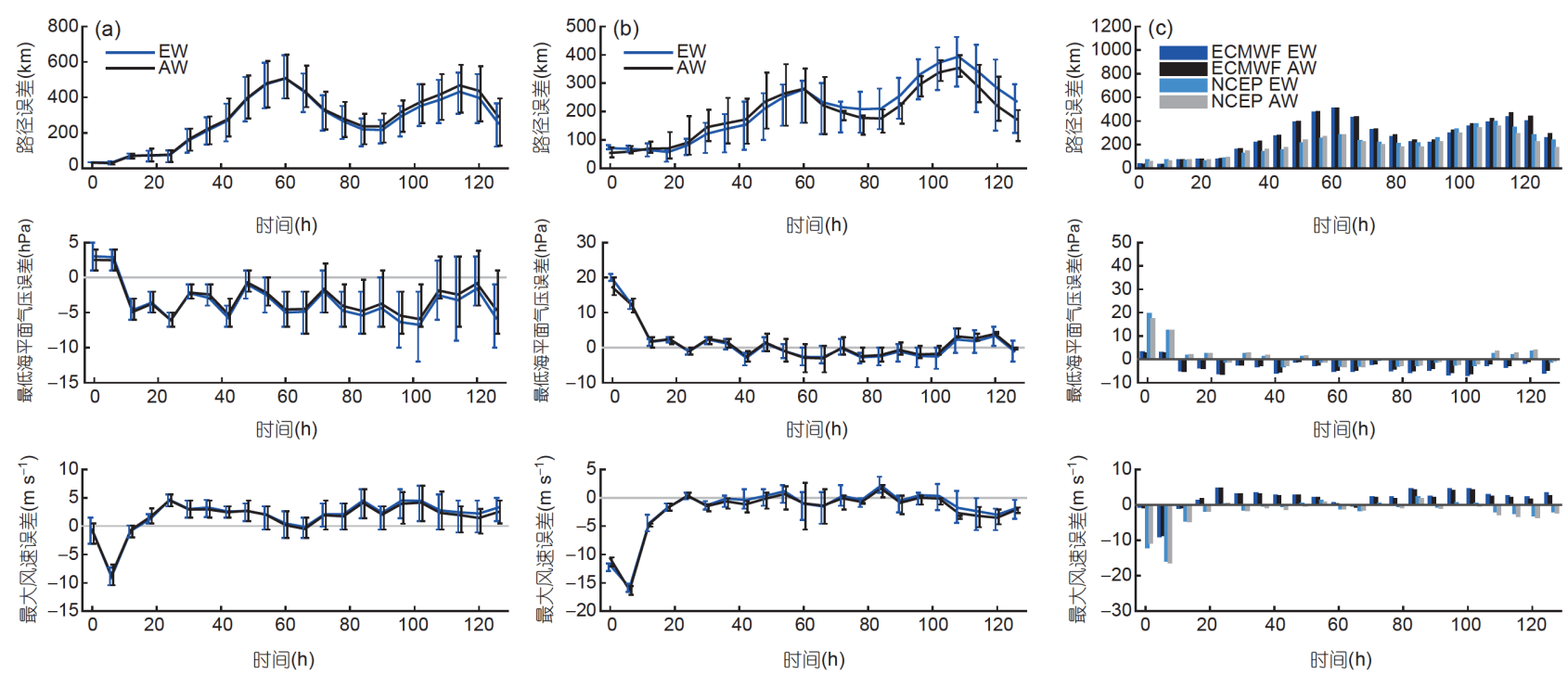

图 13 对于云雀(2018), ECMWF(a)和NCEP(b)集合预报的集合误差分布, 以及ECMWF和NCEP的集合平均误差(c)

起报时间为7月28日12:00UTC

于NCEP集合预报. 适应性权重系数的应用总体而言提 高了ECMWF和NCEP集合预报对路径和强度的预报 技巧。这是因为适应性权重通常将大于平均权重的权 重赋予预报误差较小的集合成员，因此适应性权重系 数所得的集合平均值通常较相等权重获得更小的误 差, 以及更窄的四分位数宽度. 另外, $t+p$ 时刻的预报误 差与 $t$ 时刻的预报/分析误差有正的相关(图未显示). 因 此，适应性权重系数的假设，即具有较小预报/分析误 差的成员可能得到更好的预报, 在此得到了证实. 适应 性权重系数对ECMWF集合预报的改进比对 NCEP集 合预报更为突出，这有可能是因为ECMWF集合预报 的集合数目大于NCEP集合预报。

\section{6 总结与讨论}

在2018年7 8月期间, 西北太平洋热带气旋的路径 和强度复杂多变, 对预报工作造成巨大的挑战. 三场风 暴在一个月内登陆上海十分罕见，并且热带风暴安比 (2018) 是从1949年以来第三个直接登陆上海的风暴. 因此，本文分析了形成和发展于 2018年7 8月的TC预 报技巧, 特别研究了温比亚(2018)、安比(2018)、云雀 (2018)三个登陆上海的风暴.

本文分析了ECMWF和NCEP的集合预报对TC的 预报技巧，并以 TCVitals为观测对集合预报结果进行 了验证. 为了提高集合预报的预报技巧，本文提出了
一种适应性估计集合成员权重系数的方法。适应性权 重系数的估计基于预报场和分析场相对于观测的距 离，假设当前时刻具有较小预报/分析误差的成员可能 得到未来更好的预报。适应性权重系数应用于 ECMWF和NCEP的集合预报，并与普通的相等权重系 数方法进行了比较.

首先对ECMWF和NCEP的集合预报对2018年7 8 月内 13 个 $\mathrm{TC}$ 的整体预报效果进行了分析. 根据观测位 置 $200 \mathrm{~km}$ 半径内的集合成员 (预报误差小于 $200 \mathrm{~km}$ ) 占 所有集合成员的平均比值可得，无论是强风暴还是弱 风暴，ECMWF集合预报对 $\mathrm{TC}$ 路径的预报技巧都要高 于NCEP集合预报. ECMWF和NCEP的集合预报对强 风暴路径的预报技巧高于弱风暴，这可能是由于强风 暴较弱风暴更好定义风暴中心. 强度预报误差的集合 分布结果表明，平均而言ECMWF集合预报比NCEP集 合预报具有更高的强度预报技巧. 需要注意的是, 集合 平均可能不适用于 $\mathrm{TC}$ 的强度预报，因为集合平均会导 致TC强度得到平滑．尽管ECMWF集合预报系统EPS 和NCEP全球集合预报系统GEFS使用的是不同的动力 和物理模式, ECMWF EPS比NCEP GEFS具有更高的 水平分辨率和更多的垂直层数. ECMWF和NCEP的集 合预报对强风暴的强度预报技巧要低于弱风暴，表现 为对于强风暴, ECMWF和NCEP的集合预报严重低估 了风暴强度，并且强度误差的集合分布通常会有四分 位数和尾部的偏差. 
之后分别分析了一个月内登陆上海的三个风暴的 预报技巧. 对于温比亚(2018), ECMWF和NCEP的集合 预报有相似的路径预报技巧，但ECMWF集合预报对 其强度的预报技巧略高于NCEP集合预报. NCEP集合 预报比ECMWF集合预报对安比(2018)的路径预报技 巧更高，但ECMWF集合预报比NCEP集合预报对其强 度的预报技巧更高. 对于云雀(2018)在其峰值强度时, ECMWF和NCEP的集合预报对其路径和强度的预报 技巧都较低; 当云雀(2018)减弱时, NCEP集合预报比 ECMWF集合预报具有更高的路径和强度的预报技巧.

当适应性权重系数应用于集合预报时, ECMWF 集合预报对温比亚(2018)在较短预报时效内的路径和 强度的预报技巧得到了提高. ECMWF和NCEP的集合 预报对安比(2018)的路径预报技巧得到了提高, ECMWF集合预报对其强度的预报技巧也获得了提升. 对于云雀(2018)在其峰值强度时，ECMWF和NCEP集 合预报使用适应性权重系数对其路径和强度的预报都 优于使用相等权重系数; 当云雀(2018)减弱时, 适应性 权重系数在ECMWF和NCEP集合预报中的应用也提 高了对其路径的预报技巧. 因此, 整体而言, 适应性权 重系数的应用对ECMWF和NCEP集合预报对 TC 路径 和强度的预报技巧都有改进. 适应性权重系数的优势 于ECMWF集合预报更为突出, 这可能是因为ECMWF 集合预报的集合数目大于NCEP集合预报.

适应性估计集合成员流依赖权重系数的方法应用 于集合预报得到了令人鼓舞的结果，但方法仍需进一 步的研究和讨论. 受限于数据的可用性, 本文滞后时 间 $l$ 设为 $12 \mathrm{~h}$, 但 $l$ 的取值对适应性方法性能的影响还需 进一步检验. 适应性方法在滞后和预报时长上的限制 对集合预报的影响也需要分析. 在之后的研究中, 适 应性方法在多中心集合预报和其他天气系统中的应用 也需要研究. 由于非线性系统中误差的非线性增长, 该 适应性方法可能不适用于非线性系统，因此还需要发 展能够捕捉误差快速增长的适应性方法. 另外，未来 的研究中还将对适应性方法和其他统计后处理方法进 行比较.

\section{参考文献}

Aksoy A, Aberson S D, Vukicevic T, Sellwood K J, Lorsolo S, Zhang X. 2013. Assimilation of high-resolution tropical cyclone observa- tions with an ensemble Kalman filter using NOAA/AOML/HRD's HEDAS: Evaluation of the 2008-11 vortex-scale analyses. Mon Weather Rev, 141: 1842-1865

Barkmeijer J, Buizza R, Palmer T N, Puri K, Mahfouf J F. 2001. Tropical singular vectors computed with linearized diabatic physics. Q J R Met Soc, 127: 685-708

Berner J, Shutts G J, Leutbecher M, Palmer T N. 2009. A spectral stochastic kinetic energy backscatter scheme and its impact on flowdependent predictability in the ECMWF ensemble prediction system. J Atmos Sci, 66: 603-626

Bishop C H, Shanley K T. 2008. Bayesian model averaging's problematic treatment of extreme weather and a paradigm shift that fixes it. Mon Weather Rev, 136: 4641-4652

Bonavita M, Isaksen L, Hólm E. 2012. On the use of EDA background error variances in the ECMWF 4D-Var. Q J R Meteorol Soc, 138: $1540-1559$

Buizza R. 2008. Comparison of a 51-member low-resolution ( $T_{L}$ 399L62) ensemble with a 6-member high-resolution $\left(T_{L}\right.$ 799L91) lagged-forecast ensemble. Mon Weather Rev, 136: 33433362

Cavallo S M, Torn R D, Snyder C, Davis C, Wang W, Done J. 2013. Evaluation of the advanced hurricane WRF data assimilation system for the 2009 Atlantic hurricane season. Mon Weather Rev, 141: 523-541

Davis C, Wang W, Dudhia J, Torn R. 2010. Does increased horizontal resolution improve hurricane wind forecasts? Weather Forecast, 25 : $1826-1841$

DeMaria M, Sampson C R, Knaff J A, Musgrave K D. 2014. Is tropical cyclone intensity guidance improving? Bull Amer Meteorol Soc, 95 : 387-398

Fortin V, Favre A C, Saïd M. 2006. Probabilistic forecasting from ensemble prediction systems: Improving upon the best-member method by using a different weight and dressing kernel for each member. Q J R Meteorol Soc, 132: 1349-1369

Glahn B, Peroutka M, Wiedenfeld J, Wagner J, Zylstra G, Schuknecht B, Jackson B. 2009. MOS uncertainty estimates in an ensemble framework. Mon Weather Rev, 137: 246-268

Gopalakrishnan S G, Marks Jr F, Zhang X, Bao J W, Yeh K S, Atlas R. 2011. The Experimental HWRF System: A study on the influence of horizontal resolution on the structure and intensity changes in tropical cyclones using an idealized framework. Mon Weather Rev, 139: $1762-1784$

Judt F, Chen S S, Berner J. 2016. Predictability of tropical cyclone intensity: Scale-dependent forecast error growth in high-resolution stochastic kinetic-energy backscatter ensembles. Q J R Meteorol Soc, 142: 43-57 
Kaplan J, Rozoff C M, DeMaria M, Sampson C R, Kossin J P, Velden C S, Cione J J, Dunion J P, Knaff J A, Zhang J A, Dostalek J F, Hawkins J D, Lee T F, Solbrig J E. 2015. Evaluating environmental impacts on tropical cyclone rapid intensification predictability utilizing statistical models. Weather Forecast, 30: 1374-1396

Kleist D T, Ide K. 2015. An OSSE-based evaluation of hybrid variational-ensemble data assimilation for the NCEP GFS. Part I: System description and 3D-hybrid results. Mon Weather Rev, 143: 433-451

Kurihara Y, Bender M A, Ross R J. 1993. An initialization scheme of hurricane models by vortex specification. Mon Weather Rev, 121: 2030-2045

van Leeuwen P J. 2009. Particle filtering in geophysical systems. Mon Weather Rev, 137: 4089-4114

Leutbecher M, Lock S J, Ollinaho P, Lang S T K, Balsamo G, Bechtold P, Bonavita M, Christensen H M, Diamantakis M, Dutra E, English S, Fisher M, Forbes R M, Goddard J, Haiden T, Hogan R J, Juricke S, Lawrence H, MacLeod D, Magnusson L, Malardel S, Massart S, Sandu I, Smolarkiewicz P K, Subramanian A, Vitart F, Wedi N, Weisheimer A. 2017. Stochastic representations of model uncertainties at ECMWF: State of the art and future vision. Q J R Meteorol Soc, 143: 2315-2339

Liu Q, Lord S, Surgi N, Zhu Y, Wobus R, Toth Z, Marchok T. 2006. Hurricane relocation in global ensemble forecast system. Monterey: 27th Conf. on Hurricanes and Tropical Meteorology. Amer Meteorol Soc. 5.13

Lorenz E N. 1963. Deterministic nonperiodic flow. J Atmos Sci, 20 $130-141$

Magnusson L, Bidlot J R, Bonavita M, Brown A R, Browne P A, De Chiara G, Dahoui M, Lang S T K, McNally T, Mogensen K S, Pappenberger F, Prates F, Rabier F, Richardson D S, Vitart F, Malardel S. 2019. ECMWF activities for improved hurricane forecasts. Bull Amer Meteorol Soc, 100: 445-458

Palmer T N, Buizza R, Doblas-Reyes F, Jung T, Leutbecher M, Shutts G J, Steinheimer M, Weisheimer A. 2009. Stochastic parametrization and model uncertainty. ECMWF Tech Memo, 598, 44, http://www. ecmwf.int/publications/library/ecpublications/_pdf/tm/501-600/ tm598.pdf

Raftery A E, Gneiting T, Balabdaoui F, Polakowski M. 2005. Using Bayesian Model averaging to calibrate forecast ensembles. Mon Weather Rev, 133: 1155-1174

Roulston M S, Smith L A. 2003. Combining dynamical and statistical ensembles. Tellus A, 55: 16-30
Ruf C S, Atlas R, Chang P S, Clarizia M P, Garrison J L, Gleason S, Katzberg S J, Jelenak Z, Johnson J T, Majumdar S J, O'brien A, Posselt D J, Ridley A J, Rose R J, Zavorotny V U. 2016. New ocean winds satellite mission to probe hurricanes and tropical convection. Bull Amer Meteorol Soc, 97: 385-395

Van Sang N, Smith R K, Montgomery M T. 2008. Tropical-cyclone intensification and predictability in three dimensions. Q J R Meteorol Soc, 134: 563-582

Trahan S, Sparling L. 2012. An analysis of NCEP Tropical Cyclone Vitals and potential effects on forecasting models. Weather Forecast, 27: 744-756

Typhoon Committee. 2015. Typhoon Committee Operational Manual 2015 (report). World Meteorological Organization. Retrieved November 13, 2015

Unger D A, van den Dool H, O’Lenic E, Collins D. 2009. Ensemble regression. Mon Weather Rev, 137: 2365-2379

Weng Y, Zhang F. 2016. Advances in convection-permitting tropical cyclone analysis and prediction through EnKF assimilation of reconnaissance aircraft observations. J Meteorol Soc Jpn, 94: 345358

Whitaker J S, Hamill T M. 2002. Ensemble data assimilation without perturbed observations. Mon Weather Rev, 130: 1913-1924

Yamaguchi M, Vitart F, Lang S T K, Magnusson L, Elsberry R L, Elliott G, Kyouda M, Nakazawa T. 2015. Global distribution of the skill of tropical cyclone activity forecasts on short- to medium-range time scales. Weather Forecast, 30: 1695-1709

Yamaguchi M, Nakazawa T, Hoshino S. 2012. On the relative benefits of a multi-centre grand ensemble for tropical cyclone track prediction in the western North Pacific. Q J R Meteorol Soc, 138: 2019-2029

Zhang F, Sippel J A. 2009. Effects of moist convection on hurricane predictability. J Atmos Sci, 66: 1944-1961

Zhang F, Weng Y, Sippel J A, Meng Z, Bishop C H. 2009. Cloudresolving hurricane initialization and prediction through assimilation of Doppler radar observations with an ensemble Kalman filter. Mon Weather Rev, 137: 2105-2125

Zhang Y, Meng Z, Zhang F, Weng Y. 2014. Predictability of tropical cyclone intensity evaluated through 5 -yr forecasts with a convection-permitting regional-scale model in the Atlantic Basin. Weather Forecast, 29: 1003-1023

Zhou X, Zhu Y, Hou D, Luo Y, Peng J, Wobus R. 2017. Performance of the new NCEP global ensemble forecast system in a parallel experiment. Weather Forecast, 32: 1989-2004

(责任编委: 孟智勇) 